\title{
The Current and Future of Ground and Space-VLBI Science.
}

Rudnitskiy A. G.*

Astro Space Center, Lebedev Physical Inst. RAS, Profsoyuznaya 84/32, 117997 Moscow, Russia, E-mail: almax1024@gmail.com

Likhachev S. F.

Astro Space Center, Lebedev Physical Inst. RAS, Profsoyuznaya 84/32, 117997 Moscow, Russia, E-mail: slikhacheasc.rssi.ru

Andrianov A. S.

Astro Space Center, Lebedev Physical Inst. RAS, Profsoyuznaya 84/32, 117997 Moscow, Russia, E-mail: andrianeasc.rssi.ru

Kostenko V. I.

Astro Space Center, Lebedev Physical Inst. RAS, Profsoyuznaya 84/32, 117997 Moscow, Russia, E-mail: vkostenkodasc.rssi.ru

\section{Schekinov Yu. A.}

Astro Space Center, Lebedev Physical Inst. RAS, Profsoyuznaya 84/32, 117997 Moscow, Russia,

E-mail: yuseasc.rssi.ru

Very Long Baseline Interferometry (VLBI) has been developing for more than half a century and came to a new level after the emergence of the possibility to launch radio telescope into space. Radioastron mission that is led by Astro Space Center was launched in July, 2011 and currently is the only $10-\mathrm{m}$ space radio telescope operating for more than 6 years. It operates as a SpaceEarth interferometer together with more than 40 ground telescopes, observing pulsars, masers, AGN\&QSO sources with the highest angular resolution. Millimetron is the next space observatory of Astro Space Center to be addressed for the far infrared, sub-millimeter and millimeter wavelength ranges. The unprecedented sensitivity and angular resolution of the cooled 10-m telescope, operating in a L2 orbit, will provide the astronomical community an opportunity to solve key scientific problems in astronomy and astrophysics. The observatory will have two observing modes: Space-Earth VLBI element and single dish telescope.

Frontier Research in Astrophysics - III (FRAPWS2018)

28 May - 2 June 2018

Mondello (Palermo), Italy

\footnotetext{
*Speaker.
} 


\section{Introduction}

Very Long Baseline Interferometry technique allow to reach the highest possible angular resolution for radioastronomical observations. Targets of interest include active galactic nuclei (AGN), radio galaxies, masers and pulsars.

The ground VLBI is developing rapidly and moving towards the millimeter wavelength range. For example, Event Horizon Telescope (EHT) - a global millimeter array to image the black hole shadow [1], or a high sensitivity compact millimeter array ALMA, the project that has already brought numerous number of scientific results $[2,3]$.

The senisitivy of ground VLBI is increasing with the introduction of wider bandwidths, by improving the coherence time using the water vapor radiometers, as well as phase transferring with simultaneous multi-frequency observations [4].

However, two major limitations: atmosphere influence and the finite angular resolution, constrained by the size of the Earth can be solved also by launching one of the VLBI elements to space. Such approach results into the synthesized aperture that has a size much larger than the Earth diameter providing the microarcsecond angular resolution unreachable with ground telescopes.

\section{Radioastron Mission}

On the 18th of July, 2011 from Baikonur cosmodrome was launched "Spektr-R" - a 10-meter space radio telescope - that formed the largest space-ground interferometer Radioastron [5, 6]. Radioastron is operating in a Moon-perturbed orbit. Orbit perigee varies from 400 to $65000 \mathrm{~km}$, and the apogee varies from 265000 to $360000 \mathrm{~km}$. The orbital period is about 9 days.

Currently, the mission has been operating for more than 7 years already, conducting daily space-ground VLBI observations [7]. It has four frequency bands: $0.327 \mathrm{GHz}$ (P-band), $1.665 \mathrm{GHz}$ (L-band), $4.830 \mathrm{GHz}$ (C-band) and 18.392-25.112 GHz (K-band) and is capable of observing at two bands simultaneously. Additionally, observations at K-band can be done in a multi-frequency mode (MFS) by switching in a range from 18 to $25 \mathrm{GHz}$ with $900 \mathrm{MHz}$ step. The bandwidth is 32 MHz for L-, C-, and K-band receivers and $16 \mathrm{MHz}$ for P-band.

One of the mission's peculiarities is that the space radio telescope has an onboard active hydrogen maser frequency standard (H-maser), that gives references for frequency and data sampling. The data is received by two tracking stations: 22-m radio telescope of Pushchino Radio Astronomical Observatory (PRAO) and 43-m antenna in Green Bank (NRAO, USA).

More than 40 ground telescopes are participating in the observations. All initial raw data for all observations from space and ground telescopes is collected and stored in Astro Space Center Data Processing Center [6]. This provides an ability to reprocess the raw data of any observation at any time.

The scientific program of Radioastron mission has three main directions of studies: active galactic nuclei (AGN) and quasars, masers and pulsars. AGN survey with Radioastron gave the unprecedent angular resolution at 3 frequency bands: $8 \mu$ as at K-band, $19 \mu a s$ at C-band and 104 $\mu$ as at L-band. Studies of pulsars revealed a new effects of radio emission scattering on the interstellar medium. High angular resolution of space-ground interferometer resolved the substructure in the scattering disk $[8,9]$. Later, driven by the Radioastron revealed scattering disk substructure, 
the same effect was observed for the Galactic center [10]. For the first time the detection for extra galactic maser NGC 4258 was found at extreme baseline projections up to $340000 \mathrm{~km}$ [11].

Even though, in autumn 2017, the onboard hydrogen maser has reached the end of its useful service lifetime, the mission successfully continue its operation and execution of the next observation cycle, that started in July, 2018, using the closed-loop synchronization mode [12].

There a set of scientific tasks to be solved within this observing cycle: multi-frequency polarization imaging of active galactic nuclei, monitoring of the scattering substructure for bright pulsars, observing of water fountain sources in the Galaxy, continuation of the AGN\&QSO brightness temperature monitoring with the highest angular resolution.

\subsection{Data Processing Center}

Prior the launch of Radioastron mission a dedicated Data Processing Center was developed by Astro Space Center in order to meet all the requirements for the Radioastron mission data handling and processing.

Data Processing Center can be divided into three parts: 1) online storage of 600 TB for collecting the data and offline raw data storage with the current volume of $2000 \mathrm{~TB}$ on hard drive disks and backup copy of $2000 \mathrm{~TB}$ on tapes for all raw baseband data; 2) online data storage of 80 TB and software correlator developed by Astro Space Center (ASC Correlator) to operate on high performance computing cluster of 1 Tflops for data processing; 3) 120 TB storage for processed data.

ASC Correlator was developed especially for processing the space-ground and ground VLBI data. It has a number of features: precise delay model that handle the calculation of delays for space-ground baselines, support of all known and widely used VLBI data formats, several operational modes depending on the source class (processing of maser, pulsar and AGN observations), convenient and user friendly graphical interface [12].

Currently, ASC Correlator performs processing of about $95 \%$ of all the data from Radioastron mission. By 31.08.2017 3082 out of 3351 observing sessions of the mission were processed with ASC Correlator.

\section{Millimetron Mission}

The next mission that is led by Astro Space Center will be Millimetron. It is going to be a 10-meter deployable cooled space telescope, observing at far infra-red (FIR), sub-millimeter and millimeter wavelength ranges. Millimetron will be operating in the vicinity of Lagrange point 2 (L2) located at a distance of about 1.5 million km from the Earth. There will be two operational modes: as a single dish and as space-ground interferometer. The expected payload for single dish mode are the three instruments: Millimetron Heterodyne Instrument for the Far-Infrared (MHIFI, wavelength range $60-600 \mu \mathrm{m}$ ), Short-wave Array Camera Spectrometer (SACS, camera: 4 bands: 70, 125, 230, $375 \mu \mathrm{m}$, spectrometer: long slit grating spectrometers: 50 - $450 \mu \mathrm{m}$ ), Long waveArray Camera Spectrometer (LACS, camera: 4 bands: 0.4, 0.7, 1.2, $2.3 \mathrm{~mm}$, FTS spectrometer: 0.3 - $3 \mathrm{~mm}$ ). Space-ground VLBI is expected to have a set of receivers that will cover the wavelength range of $1-17 \mathrm{~mm}$. 
Aperture of 10-meters for the single dish mode will provide a capability to resolve more than $90 \%$ of the sources in the cosmic infrared background (CIB). At the same time, the expected resolution in the interferometric mode will be 100 times better than it was for Radioastron mission.

Conception of the scientific program of the mission is to have 5-6 breakthrough scientific tasks to be solved. The following problems are to be considered as the candidates for the scientific program:

1) Determination of the nature of supermassive bodies located in the centers of our Galaxy, M31 (Andromeda) and M87;

2) Search for wormholes in nearby galaxies with supermassive central objects using the Millimetron;

3) Measurement of the CIB spectrum distortions;

4) Measurements of the interstellar dust emission contribution at high galactic latitudes;

5) Direct observations of biomolecules in the atmospheres of transit exoplanets;

6) Search for life precursor signs in the planetary and star formation regions;

7) The origin of water in the habitable zones of planetary systems;

Millimetron project is included in Russian Federal Space Program with the following full financial support from Russian state corporation "ROSCOSMOS". It is expected that Millimetron spacecraft will be finished till 2025 with the further launch in one-two years.

\subsection{Millimetron Mission Support Center}

For Millimetron a separate Mission Support Center will be organized and it will cover all the tasks required for ground support:

1) Collecting and archiving of the data;

2) Processing of all the observational data and organizing the information exchange among mission's participants;

3) Navigational support of the mission: orbit simulations, measurements and reconstruction.

Comparing to Radioastron mission Millimetron applies stronger requirements for data processing and collection. The expected volume of data $3300 \mathrm{~PB} / \mathrm{year}$ or $33000 \mathrm{~PB}$ for 10 years of operation, which mostly come from the space-ground VLBI observations.

\section{Conclusion}

Radioastron mission gave a great experience in the development of large space radio telescopes, organizing and conducting large scientific programs and surveys, setting up a VLBI data delivery and its pipeline processing, as well as allowed to get the new scientific results by providing the highest angular resolution in the joint space-ground VLBI observations.

This experience made it possible to outline the main critical points of any space-ground VLBI mission: 1) Accuracy of the orbit determination: use of laser ranging and selective VLBI tracking of the spacecraft can dramatically improve the accuracy of orbit determination; 2) On-board accelerometer and clock: devices to be selected according to the requirements for space craft acceleration and velocity; 3) Choice of the baseline vector projection to avoid "gaps" on the (u,v)-plane, as well as the accurate scheduling of the mission scientific program. It's possible that successive 
scientific targets will be rare enough; 4) Provide acceptable sensitivity; 5) Provide a consistent data downlink channel supply.

Millimetron will be the next step of space based astronomy and will use all the experience that was gained during the operation of Radioastron. As a single dish and space-ground VLBI observatory in $\mathrm{mm}$, sub-mm and FIR it will provide unprecedented sensitivity and the highest angular resolution to solve the most breaking through astrophysical scientific tasks.

\section{References}

[1] Falcke, H., Melia, F., Agol, E., Viewing the Shadow of the Black Hole at the Galactic Center, Astrophysical Journal Letters, 528, L13-L16, 2000.

[2] Gorman, E. O., Kervella P., Harper G. M., Richards A. M. S., Decin L., Montarges M., McDonald I., The inhomogeneous sub-millimeter atmosphere of Betelgeuse, Astronomy\&Astrophysics, 602, L10, 2017.

[3] Stephens, I. W., Looney, L. W., Kwon, W., FernÃąndez-LÃşpez, M., Hughes, A. M., Mundy, L. G., Crutcher, R. M., Li, Zhi-Yun, Rao, R. Spatially resolved magnetic field structure in the disk of a $T$ Tauri star, 514, 597, 2014.

[4] Zhao, G.-Y., Algaba, J. C., Lee, S. S., Jung, T., Dodson, R., Rioja, M., Byun, D.-Y., Hodgson, J., Kang, S., Kim, D.-W., Kim, J.-Y., Kim, J.-S., Kim, S.-W., Kino, M., Miyazaki, A., Park, J.-H., Trippe, S., Wajima, K., The Power of Simultaneous Multi-frequency Observations for mm-VLBI: Beyond Frequency Phase Transfer, Astronomical Journal, 155, 26, 2018.

[5] Kardashev, N. S., Radioastron: a radio telescope many times the size of Earth. Research program., Physics Uspekhi, 52, Issue 11, pp. 1127-1137, 2009.

[6] Kardashev, N. S., Khartov, V. V., Abramov, V. V., Avdeev, V. Yu., et al., "RadioAstron" - A telescope with a size of $300000 \mathrm{~km}$ : Main parameters and first observational results, Astronomy Reports, 57 , Issue 3, pp. 153-194, 2013.

[7] Kardashev, N. S., Alakoz, A. V., Andrianov, A. S., Artyukhov, M. I., Baan, W., et. al., RadioAstron Five Years After Launch: Main Science Results, Solar System Research, 51, 535, 2017.

[8] Gwinn, C. R., Popov, M. V., Bartel, N., Andrianov, A. S., Johnson, M. D., Joshi, B. C., Kardashev, N. S., et. al., PSR B0329+54: Statistics of Substructure Discovered within the Scattering Disk on RadioAstron Baselines of up to 235,000 km, The Astrophysical Journal, Volume 822, Issue 2, article id. 96, 13 pp., 2016.

[9] Popov, M. V., Bartel, N., Gwinn, C. R., Johnson, M. D., Andrianov, A., Fadeev, E., Joshi, B. C., Kardashev, N., PSR B0329+54: substructure in the scatter-broadened image discovered with RadioAstron on baselines up to $330000 \mathrm{~km}$, Monthly Notices of the Royal Astronomical Society, Volume 465, Issue 1, p.978-985, 2017.

[10] Gwinn, C. R., Kovalev, Y. Y., Johnson, M. D., Soglasnov, V. A., Discovery of Substructure in the Scatter-broadened Image of Sgr A*, Astrophysical Journal Letters, Volume 794, Issue 1, L14, 2014

[11] Baan, W., Alakoz, A., An, Tao, Ellingsen, S., Henkel C., Imai, H., Kostenko, V., Litovchenko, I., Moran, J., Sobolev, A., Tolmachev, A., H2O MegaMasers: a RadioAstron success story, eprint arXiv:1801.08796, 2018. 
[12] Likhachev, S. F., Kostenko, V. I., Girin, I. A., Andrianov, A. S., Rudnitskiy, A. G., Zharov, V. E., Software Correlator for Radioastron Mission, Journal of Astronomical Instrumentation, Volume 6, Issue 3, id. 1750004-131, 2017.

\section{DISCUSSION}

JIM BEALL QUESTION: Do you have an estimation of the accuracy of orbit determination required for Space-Ground VLBI?

ALEXEY RUDNITSKIY ANSWER: With laser ranging methods, the estimated orbit accuracy could reach dozens of centimeters in position estimation and for the velocity of dozen $\mathrm{mm} / \mathrm{s}$. 